\title{
Strategic Valuation of Smart Grid Technology Options in Distribution Networks
}

\author{
Ioannis Konstantelos, Member, IEEE, Spyros Giannelos, Student Member, IEEE, and Goran Strbac, \\ Member, IEEE
}

\begin{abstract}
The increasing penetration of renewable distributed generation (DG) sources in distribution networks can lead to violations of network constraints. Thus, significant network reinforcements may be required to ensure that DG output is not constrained. However, the uncertainty around the magnitude, location and timing of future DG capacity renders planners unable to take fully-informed decisions and integrate DG at a minimum cost. In this paper we propose a novel stochastic planning model that considers investment in conventional assets as well as smart grid assets such as demandside response, coordinated voltage control and soft open points (SOPs). The model also considers the possibility of active power generation curtailment of the DG units. A node-variable formulation has been adopted to relieve the substantial computational burden of the resulting mixed integer non-linear programming (MINLP) problem. A case study shows that smart technologies can possess significant strategic value due to their inherent flexibility in dealing with different system evolution trajectories. This latent benefit remains undetected under traditional deterministic planning approaches which may hinder the transition to the smart grid.

Index Terms - coordinated voltage control (CVC), demand side response (DSR), soft-open point (SOP), strategic value, stochastic optimization.
\end{abstract}

\section{NOMENCLATURE}

Sets and indices

$\Omega_{C} \quad$ Set of normally-open points, indexed $c$

$\Omega_{D G} \quad$ Set of DG units, indexed $g$

$\Omega_{E} \quad$ Set of epochs, indexed $e$

$\Omega_{L} \quad$ Set of distribution lines, indexed $l$

$\Omega_{M} \quad$ Set of scenario tree nodes, indexed $m$

$\Omega_{N} \quad$ Set of system buses, indexed $n$

$\Omega_{T H} \quad$ Set of thermal units \& substations, indexed $g$

$\Omega_{Q} \quad$ Set of typical days, indexed $q$

$\Omega_{T_{q}} \quad$ Set of demand periods corresponding to $q$, indexed $t$

$\varepsilon_{m} \quad$ Epoch to which scenario tree node $m$ belongs

$\Phi_{k}(m)$ Time-ordered set containing all parent nodes of node $m$, from the first epoch up to epoch $\varepsilon_{m}-k$

\section{Input Parameters}

$\gamma_{x} \quad$ Investment cost (£/year) for reinforcing line $l$ $(x=B)$, for CVC $(x=C)$, for $\operatorname{DSR}(x=D)$,

I. Konstantelos, S. Giannelos and G. Strbac are with the Control and Power Group, Faculty of Electrical and Electronic Engineering, Imperial College London, UK. for $\operatorname{SOP}(x=S)$

$\delta_{t} \quad$ Duration of one period (hours).

$\lambda \quad$ Consumer Payments for DSR flexibility $€ /(k W h)$

$\eta_{f} \quad$ SOP efficiency in transporting active power $(\%)$

$\pi_{m} \quad$ Probability of scenario-tree node $m$ occurring

$\Psi_{n, t} \quad$ Tangent of the load angle at bus $n$ at period $t$

$\zeta_{t, g}$ Time series representing the intermittency of the renewable DG unit $g$, expressed as a percentage of installed capacity.

$b_{l}^{\mathrm{A}} \quad$ Line susceptance before reconductoring (pu)

$b_{l}^{\mathrm{N}} \quad$ Line susceptance after reconductoring $(\mathrm{pu})$

$c^{c} \quad$ Cost of curtailing DG output $(£ / k W h)$

$\bar{D}_{t, n} \quad$ Max load that can be shifted to $t$ at bus $n(k W)$

$d_{t, n} \quad$ Real power demand at bus $n$, period $t(k W)$

$F_{l} \quad$ Initial capacity of line $l(k W)$

$F_{\max }$ Extra capacity, obtained from reconductoring relative to the existing capacity $(k W)$

$f_{t, n} \quad$ Percentage of the initial load that is available, at bus $n$ at period $t$, for shifting to a period $\tau \neq t$

$g_{l}^{\mathrm{A}} \quad$ Line conductance before reconductoring $(\mathrm{pu})$

$g_{l}^{N} \quad$ Line conductance after reconductoring (pu)

$I_{n, g} \quad$ Equals 1 if $g$ is connected to bus $n$, else it equals 0

$k_{x} \quad$ Build time (epochs) for reconductoring $(x=L)$, for $\operatorname{CVC}(x=C), \operatorname{DSR}(x=D), \operatorname{SOP}(x=S)$

$N_{q} \quad$ Times of occurrence of day $q$ in a year

$n_{c}^{x} \quad$ Primary $(x=a)$ and secondary $(x=b)$ terminals of SOP installed at $c$.

$P_{m, g}^{\max } \quad$ Max real power stable generation of $g(k W)$

$P_{c}^{\max }$ Real power capacity of SOP installed at $c(k W)$

$Q_{c}^{\max }$ Reactive capacity of SOP installed at $c(k V A r)$

$r_{\varepsilon_{m}}^{I} \quad$ Cumulative discount factor for investment cost

$r_{\varepsilon_{m}}^{O} \quad$ Cumulative discount factor for operational cost

$S_{m, g}^{\max }$ Installed capacity of $g \in \Omega_{T H} U \Omega_{D G}$ at $m(k V A)$

$u_{l} \quad$ Sending bus of line $l$

$v_{l} \quad$ Receiving bus of line $l$

$V_{\text {set }}$ Voltage target value at the AVC relay of the substation OLTC transformer at node $m(\mathrm{pu})$

$V_{\min }$ Minimum voltage statutory limit (pu)

$V_{\max }$ Maximum voltage statutory limit (pu)

$V_{\min }^{c v c}$ Minimum voltage attainable by CVC (pu)

$V_{\max }^{c v c}$ Maximum voltage attainable by $\mathrm{CVC}(\mathrm{pu})$

\section{Decision Variables}

$\theta_{m, t, n} \quad$ Voltage angle corresponding to bus $n$ (rad)

$\xi_{m, t, n}^{d}$ Load at $m, n$ shifted away from period $t(k W)$ 
$\xi_{m, t, n}^{c}$ Load at $m, n$ shifted to period $t(k W)$

$\omega_{m}^{x} \quad$ Investment $(x=I)$ or operational $(x=O)$ cost $(£)$

$B_{m, l} \quad$ Binary variable for deciding to reconductor $l$ at $m$

$\tilde{B}_{m, l} \quad$ State variable corresponding to $B_{m, l}$

$C_{m} \quad$ Binary variable for deciding to invest in CVC at $m$

$\tilde{C}_{m} \quad$ State variable corresponding to $C_{m}$

$D_{m, n}$ Binary variable for deciding to invest DSR at $m, n$

$\widetilde{D}_{m, n} \quad$ State variable corresponding to $D_{m, n}$

$F_{m, l} \quad$ Continuous variable for the extra capacity due to reconductoring $l$ at $m(k W)$

$\tilde{F}_{m, l} \quad$ State variable corresponding to $F_{m, l}$

$G_{m, t, c}$ Real power drawn by SOP at terminal $n_{c}^{a}(k W)$

$H_{m, t, c, n}^{Q}$ Reactive power drawn by SOP at bus $n(k V A r)$

$P_{m, t, g}$ Real power output of unit $g$ at $m, t(k W)$

$P_{m, t, l}^{s} \quad$ Real power flow at sending bus of $l$ at $m, t(k W)$

$P_{m, t, l}^{r} \quad$ Real power flow at receiving bus of $l$ at $m, t(k W)$

$Q_{m, t, g}$ Reactive power output of $g$ at $m, t(k V A r)$

$Q_{m, t, l}^{s}$ Reactive flow at sending bus of $l$ at $m, t(k V A r)$

$Q_{m, t, l}^{r} \quad$ Reactive flow at receiving bus of $l$ at $m, t(k V A r)$

$S_{m, c} \quad$ Binary variable for SOP investment decision at $m, c$

$\tilde{S}_{m, c} \quad$ State variable corresponding to $S_{m, c}$

$V_{m, t, n} \quad$ Voltage magnitude at bus $n$ at $m, t$ (pu)

$V_{m, t}^{C V C} \quad$ Substation voltage target regulated by CVC (pu)

$V_{m, t}^{n o C}$ Substation voltage target in absence of CVC (pu)

\section{INTRODUCTION}

$\mathrm{P}$ ENETRATION of renewable DG sources is set to increase worldwide over the coming decades as part of the global decarbonisation effort. As a result, distribution networks are facing challenges related to increased peaks and undesirable voltage excursions in the event of high net DG production levels. It follows that significant network reinforcements may be required over the next decades to facilitate this transition. However, a very significant additional challenge in realizing this transition is the increased uncertainty that surrounds future generation developments, preventing network planners from making fully informed decisions. This is aggravated by reduced commissioning times for DG sources, rendering anticipatory planning the only viable option for the timely accommodation of new entrants, while inadvertently giving rise to the prospect of inefficient investments and stranded assets. In response to the potentially large volume of new DG sources to be connected as well as the high uncertainty that surrounds timing, magnitude and location of connection, new planning frameworks are needed for cost-efficient strategic investments.

Historically, distribution networks have been based on unidirectional power flows and designed to accept bulk power from the transmission network and distribute it to customers. Supported by energy policy mechanisms, penetration of DG is drastically increasing, which is changing power flow patterns in distribution networks. In general, the ability of the network to accommodate DG is determined by its voltage and thermal limits. The first pertains to the fact that bus voltages may rise beyond statutory limits at times of low demand and high output of DG units. The latter pertains to events of high DG feed-in, resulting in high current flows beyond the line and transformer thermal limits. Under the current EU legislation, Distribution Network Operators (DNOs) provide firm connections to new DG. However, a review of grid access regimes with a shift to non-firm connections is becoming increasingly relevant in order to allow cost-effective solutions to deal with network constraints [1]. Given that in many areas distribution networks are already close to their DG hosting limit, significant network reinforcements are required to alleviate voltage and thermal constraints. Most importantly, since DG installations proceed without prior coordination with the network planners, it is not possible for DNOs to accurately determine in advance where voltage and/or thermal violations may occur. As a result, conventional network reinforcements run the risk of asset stranding, thus potentially limiting the effectiveness and rate of DG deployment due to increased integration costs.

Active control of bus voltages and the demand-supply balance through power electronics and other smart technologies is an alternative to conventional reinforcements [2]. Schemes like coordinated voltage control (CVC) [3], softopen point (SOP) [4], demand-side response (DSR) [5], [6] and active power generation curtailment (APGC) of a DG unit [1] can assist in relieving parts of the system from increased DG output. These technologies can also support the planner in managing the risk of stranded conventional assets by constituting interim investment options that enhance the utilization of existing assets and defer large capital commitments on a conditional basis until a scenario realization suggests they would be economically justified. This feature becomes apparent when examining strategic investment under uncertainty. Although the importance of building future-proof energy infrastructure in view of the increasing uncertainty has been recognized by governments and institutions worldwide (e.g. [7]), little work has been carried out on how smart grid technologies can assist in this task.

In this context, this paper focuses on distribution network planning under uncertainty. We demonstrate that smart grid technologies constitute valuable options for enabling cost effective integration of DG under uncertainty. In addition, we show that deterministic approaches can systematically undervalue the flexibility that such assets provide; traditional investment valuation techniques are biased towards premature commitment and can pose a significant barrier to the advent of the flexible smart grid paradigm. To this end, a novel multistage stochastic planning framework is proposed and the concept of the strategic value of smart grid technologies is introduced. The increased computational burden of the developed model due to the presence of binary variables and the non-linear ACOPF formulation is managed by employing a node-variable formulation. The contributions of this paper can be summarized as follows:

- Formulation of a stochastic planning methodology for obtaining the strategic value of a portfolio of different smart grid technologies. The developed methodology 
allows the accommodation of different sources of uncertainty described via a scenario tree.

- Presentation of mathematical formulations for operating and investing in SOP, DSR, CVC assets in distribution networks.

- Demonstration of the strategic 'wait-and-see' flexibility that smart technologies can provide as well as their exclusion when uncertainty is ignored, highlighting the shortcomings of deterministic planning standards.

- Showcase of the aforementioned concepts through a case study focusing on the uncertainty regarding the size and location of future distributed PV units in a medium-voltage network.

The remaining sections of this paper are organized as follows: Section II reviews the existing literature and gives insights regarding decision-making under uncertainty. Section III presents the problem's mathematical formulation. Section IV demonstrates the application of the proposed planning framework to an $11 \mathrm{kV}$ distribution network. Section V provides conclusions and recommendations for future work.

\section{StRAtegic VAlue of SMART GRID TechnOlogieS}

Distribution planning under uncertainty is an active research topic, with most publications traditionally focusing on deterministic network design frameworks [8], [9], [10]. Furthermore, in most existing models the deployment pattern of DG sources is regarded either as an input parameter or as a variable to be optimized [11], [12]. Although integrated resource planning would lead to efficient system development, in many jurisdictions there is no coordination between DG deployment and network investment; in such cases future connection patterns should be modeled as stochastic parameters. Recently, there have been efforts to consider the dynamic investment problem under uncertainty through Real Options analysis [13]. Approaches such as this, which aim to capture the network planner's flexibility in addressing uncertainty, are becoming increasingly relevant. The application scope of such valuation frameworks is limited to a small number of candidate investment strategies defined a priori. However, in reality, a large number of strategic opportunities can arise in all irreversible dynamic decision processes due to the inter-temporal resolution of uncertainty and the possibility for managerial flexibility. This is certainly the case for distribution network planning, which entails decisions with respect to numerous asset types and possible investment timings. To this end, the use of optimization is essential for the identification of the optimal investment strategy across all possible investment combinations [14].

As we demonstrate in this paper, when examined in an uncertainty setting, smart technologies can provide system benefits beyond those detected under deterministic studies. This latent value stems from their flexibility to meet adverse scenario realizations without prematurely committing to large upgrades until necessary. More specifically, smart technologies are characterized by two types of flexibility. First of all, most smart technologies may have broader nonlocalized effects, meaning that investment is not targeted at improving DG hosting capability of a single line or busbar but rather aims at enhancing the utilization of existing assets in a larger network area by improving controllability. As a result, opting for smart grid solutions can provide a natural hedge when future DG deployment patterns are characterized by locational uncertainty. Secondly, smart technologies typically have faster commissioning times than conventional reinforcements as lengthy planning permissions, asset reinforcement activities and public works can be avoided. Thus, the ability for deployment of smart assets on a conditional basis can render 'wait-and-see' investment strategies cost-effective and viable.

The planner's ability to rely on smart technologies as interim solutions can have a significant impact on the chosen investment strategy as it can allow the deferral of conventional reinforcement, thereby reducing the system costs. To describe this latent benefit of smart grid technologies, we choose to use the term strategic value [14], [15]. Although much has been written about the importance of incorporating strategic value in investment appraisals and the concept is gaining traction with industry and institutions worldwide (see, for example, [16], [17]), the proposed methodology formalizes the effort to fully quantify the value of smart technologies when facing uncertainty. In the following sections we show that this strategic value, which is ignored under deterministic approaches, can be significant in size and can tip investment in favor of smart grid investments when taken into account in the planning process.

\section{MATHEMATICAL FORMULATION}

The planning problem is formulated as a mixed integer non-linear problem (MINLP), where binary variables are used to denote investment decisions. In particular, the planner has the choice to invest in reconductoring (conventional investment) and in smart technologies (CVC, DSR, and SOP). According to the nomenclature, the decision for investment in CVC, DSR, and SOP is denoted by the binary variables $C_{m}$, $D_{m, n}$, and $S_{m, c}$ respectively. In addition, a non-linear ACOPF formulation has been adopted to capture both thermal and voltage constraints [18].

Uncertainty is modelled in the form of a multi-stage scenario tree of $\left|\Omega_{\mathrm{M}}\right|$ nodes spanning $\left|\Omega_{\mathrm{E}}\right|$ epochs (also referred to as stages) capturing the possible system states across the planning horizon. Each node represents a multi-year period over which the system state remains unchanged. The cumulative discount factors $r_{\varepsilon_{m}}^{I}$ and $r_{\varepsilon_{m}}^{O}$ are used to appropriately weight investment and operation costs incurred in each node $m$ in terms of the time value of money and epoch duration. For example, $r_{\varepsilon_{m}}^{I}$ accounts for the fact that annual capital payments are to be made from the year an investment decision is made until the final year of the study. The mathematical formulation presented below is of the nodevariable type [20]. As such, each scenario-specific decision variable has an associated index $m$, denoting the scenario tree node to which it pertains as well as the corresponding stage (denoted by $\varepsilon_{m}$ ). In terms of investment, relevant state 
variables and equations are introduced to communicate decisions taken at a node $m$ to all subsequent children nodes, thus enforcing the scenario tree structure. As demonstrated in [21], node-variable formulations can result in significant computational benefits compared to their scenario-variable counterparts due to eliminating redundant variables and removing the need for non-anticipativity constraints.

The focus of the presented formulation is long-term uncertainty related to the evolution of the system, such as deployment of DG. Uncertainties related to the operational timescale, such as demand and renewables output, are not considered. Given that the majority of operational decisions being considered can be regarded as automatic and almost instantaneous (e.g. operation of CVC or curtailment of renewable output) relevant operational decisions can be optimally drawn on the basis of the eventual demand and renewable output realization. In other words, uncertainty at the operational timescale does not impact control decisions. DSR constitutes an exception to this since its operation involves shifting energy between different hours of the day and can thus suffer from inaccurate demand and/or generation forecasts. However, in this research we assume that the demand pattern is fully known a priori; modelling stochastic DSR operation in a multi-stage planning setting poses significant computational challenges that are beyond the scope of this paper. A set of typical days $\Omega_{Q}$ is used to capture demand and resource variability throughout a year. Clustering analysis techniques can be applied on historical data to obtain representative time-series that preserve temporal and spatial correlations, as proposed in [19].

The planner has the choice to invest in reconductoring (conventional investment) and in smart technologies (CVC, DSR, and SOP). Note also that DSR can be deployed at any load bus, SOPs can be installed at any normally-open point (NOP), and CVC can be installed at the substation. The planner can also optimally perform APGC which involves paying the DG owners for curtailing their output. The presented model is formulated on the basis of generic renewable units capable of instantaneous curtailment of their active power output. More specific operational requirements dictated by a jurisdiction's grid code can also be accommodated with straightforward modifications.

The network is not operated in a microgrid fashion. Rather, it is connected through a $33 / 11 \mathrm{kV}$ substation to the main grid. The substation is modelled as a slack busbar (denoted by $G_{1}$ ) which can generate or absorb power in order to balance the system. For simplicity, the capacity is assumed to always be sufficiently large and thus not constrain operation. Naturally, the DG units have priority in power generation over $G_{1}$ due to the cost of curtailing DG output. The problem's mathematical formulation is as follows.

$$
\begin{aligned}
& z=\min \left\{\sum_{m \in \Omega_{M}} \pi_{m}\left(r_{\varepsilon_{m}}^{I} \omega_{m}^{I}+r_{\varepsilon_{m}}^{o} \omega_{m}^{o}\right)\right\} \\
& \omega_{m}^{I}=C_{m} \gamma_{C}+\sum_{l \in \Omega_{L}} B_{m, l} \gamma_{B}+\sum_{n \in \Omega_{N}} D_{m, n} \gamma_{D}+\sum_{c \in \Omega_{C}} S_{m, c} \gamma_{S}
\end{aligned}
$$

$$
\begin{aligned}
& \omega_{m}^{o}=\sum_{\substack{q \in \Omega_{Q} \\
t \in \Omega_{T q} \\
g \in \Omega_{D G}}} N_{q} \delta_{t} c^{c}\left(P_{m, g}^{\max } \zeta_{t, g}-P_{m, t, g}\right)+\sum_{\substack{q \in \Omega_{Q} \\
t \in \Omega_{T_{q}} \\
n \in \Omega_{N}}} N_{q} \delta_{t} \xi_{m, t, n}^{d} \lambda \\
& \left(P_{m, t, g}\right)^{2}+\left(Q_{m, t, g}\right)^{2} \leq\left(S_{m, g}^{\max } \zeta_{t, g}\right)^{2} \quad \forall m, t, g \Omega_{T H} U \Omega_{D G} \\
& \tilde{F}_{m, l}=\sum_{\varphi \in \Phi_{k_{L}}(m)} B_{\varphi, l} F_{\max } \\
& \tilde{B}_{m, l}=\sum_{\varphi \in \Phi_{k_{L}}(m)} B_{\varphi, l} \\
& \tilde{C}_{m}=\sum_{\varphi \in \Phi_{k_{C}}(m)} C_{\varphi} \\
& \widetilde{D}_{m, n}=\sum_{\varphi \in \Phi_{k_{D}}(m)} D_{\varphi, n} \\
& \tilde{S}_{m, c}=\sum_{\varphi \in \Phi_{k_{S}}(m)} S_{\varphi, c} \\
& P_{m, t, l}^{s}=\left(1-\widetilde{B}_{m, l}\right)\left[V_{m, t, u_{l}}^{2} g_{l}^{A}-V_{m, t, u_{l}} V_{m, t, v_{l}} g_{l}^{A} .\right. \\
& \left.\cos \left(\theta_{m, t, u_{l}}-\theta_{m, t, v_{l}}\right)-V_{m, t, u_{l}} V_{m, t, v_{l}} b_{l}^{A} \sin \left(\theta_{m, t, u_{l}}-\theta_{m, t, v_{l}}\right)\right] \\
& +\widetilde{B}_{m, l}\left[V_{m, t, u_{l}}^{2} g_{l}^{N}-V_{m, t, u_{l}} V_{m, t, v_{l}} g_{l}^{N} \cos \left(\theta_{m, t, u_{l}}-\theta_{m, t, v_{l}}\right)\right. \\
& \left.-V_{m, t, u_{l}} V_{m, t, v_{l}} b_{l}^{N} \cdot \sin \left(\theta_{m, t, u_{l}}-\theta_{m, t, v_{l}}\right)\right] \quad \forall m, t, l \\
& P_{m, t, l}^{r}=\left(1-\widetilde{B}_{m, l}\right)\left[V_{m, t, v_{l}}^{2} g_{l}^{A}-V_{m, t, v_{l}} V_{m, t, u_{l}} g_{l}^{A} .\right. \\
& \left.\cos \left(\theta_{m, t, v_{l}}-\theta_{m, t, u_{l}}\right)-V_{m, t, u_{l}} V_{m, t, v_{l}} b_{l}^{A} \sin \left(\theta_{m, t, v_{l}}-\theta_{m, t, u_{l}}\right)\right] \\
& +\widetilde{B}_{m, l}\left[V_{m, t, v_{l}}^{2} g_{l}^{N}-V_{m, t, u_{l}} V_{m, t, v_{l}} g_{l}^{N} \cos \left(\theta_{m, t, v_{l}}-\theta_{m, t, u_{l}}\right)\right. \\
& \left.-V_{m, t, u_{l}} V_{m, t, v_{l}} b_{l}^{N} \cdot \sin \left(\theta_{m, t, v_{l}}-\theta_{m, t, u_{l}}\right)\right] \\
& Q_{m, t, l}^{s}=\left(1-\widetilde{B}_{m, l}\right)\left[-V_{m, t, u_{l}}^{2} b_{l}^{A}-V_{m, t, u_{l}} V_{m, t, v_{l}} g_{l}^{A} .\right. \\
& \left.\sin \left(\theta_{m, t, u_{l}}-\theta_{m, t, v_{l}}\right)+V_{m, t, u_{l}} V_{m, t, v_{l}} b_{l}^{A} \cos \left(\theta_{m, t, u_{l}}-\theta_{m, t, v_{l}}\right)\right] \\
& +\widetilde{B}_{m, l}\left[-V_{m, t, u_{l}}^{2} b_{l}^{N}-V_{m, t, u_{l}} V_{m, t, v_{l}} g_{l}^{N} \sin \left(\theta_{m, t, u_{l}}-\theta_{m, t, v_{l}}\right)\right. \\
& \left.+V_{m, t, u_{l}} V_{m, t, v_{l}} b_{l}^{N} \cos \left(\theta_{m, t, u_{l}}-\theta_{m, t, v_{l}}\right)\right] \\
& \forall m, t, l(12) \\
& Q_{m, t, l}^{r}=\left(1-\widetilde{B}_{m, l}\right)\left[-V_{m, t, v_{l}}^{2} b_{l}^{A}-V_{m, t, u_{l}} V_{m, t, v_{l}} g_{l}^{A} .\right. \\
& \left.\sin \left(\theta_{m, t, v_{l}}-\theta_{m, t, u_{l}}\right)+V_{m, t, u_{l}} V_{m, t, v_{l}} b_{l}^{A} \cos \left(\theta_{m, t, v_{l}}-\theta_{m, t, u_{l}}\right)\right] \\
& +\widetilde{B}_{m, l}\left[-V_{m, t, v_{l}}^{2} b_{l}^{N}-V_{m, t, u_{l}} V_{m, t, v_{l}} g_{l}^{N} \sin \left(\theta_{m, t, v_{l}}-\theta_{m, t, u_{l}}\right)\right. \\
& \left.+V_{m, t, u_{l}} V_{m, t, v_{l}} b_{l}^{N} \cos \left(\theta_{m, t, v_{l}}-\theta_{m, t, u_{l}}\right)\right] \quad \forall m, t, l \\
& \left(P_{m, t, l}^{s}\right)^{2}+\left(Q_{m, t, l}^{s}\right)^{2} \leq\left[F_{l}+\tilde{F}_{m, l}\right]^{2} \\
& \left(P_{m, t, l}^{r}\right)^{2}+\left(Q_{m, t, l}^{r}\right)^{2} \leq\left[F_{l}+\tilde{F}_{m, l}\right]^{2} \\
& V_{\min } \leq V_{m, t, n} \leq V_{\max } \\
& V_{m, t, 1}=V_{m, t}^{c v c}+V_{m, t}^{n o c} \\
& V_{\min }^{c v c} \cdot \tilde{C}_{m} \leq V_{m, t}^{c v c} \leq V_{\max }^{c v c} \cdot \tilde{C}_{m} \\
& V_{m, t}^{n o c}=V_{\text {set }_{m}} \cdot\left(1-\tilde{C}_{m}\right) \\
& \sum_{t \in \Omega_{T_{q}}}\left(\xi_{m, t, n}^{d}-\xi_{m, t, n)}^{c}=0\right. \\
& \xi_{m, t, n}^{d} \leq \widetilde{D}_{m, n} \cdot f_{t, n} \cdot d_{t, n} \quad \forall m, t, n \\
& \xi_{m, t, n}^{c} \leq \widetilde{D}_{m, n} \bar{D}_{t, n} \quad \forall m, t, n \\
& G_{m, t, c} \leq P_{c}^{\max } \cdot \tilde{S}_{m, c} \quad \forall m, t, c \\
& \left|H_{m, t, c, n}^{Q}\right| \leq Q_{c}^{\max } \cdot \tilde{S}_{m, c} \quad \forall m, t, c \\
& \forall m, t, l(14) \\
& \forall m, t, n-\{1\} \text { (16) } \\
& \forall m, t \quad(17) \\
& \forall m, t \text { (18) } \\
& \forall m, t \text { (19) } \\
& \forall m, n, q \quad(20)
\end{aligned}
$$

$$
\begin{aligned}
& \sum_{g \in \Omega_{D g} U \Omega_{T H}} P_{m, t, g} I_{n, g}-\sum_{l \in\left\{\Omega_{L} \mid v_{l}=n\right\}} P_{m, t, l}^{r}-\sum_{l \in\left\{\Omega_{L} \mid u_{l}=n\right\}} P_{m, t, l}^{s}= \\
& +d_{t, n}-\xi_{m, t, n}^{d}+\xi_{m, t, n}^{c}+\sum_{c \in\left\{\Omega_{C} \mid n=n_{c}^{a}\right\}}\left(G_{m, t, c}\right)
\end{aligned}
$$




$$
\begin{aligned}
& +\sum_{c \in\left\{\Omega_{C} \mid n=n_{c}^{b}\right\}}\left(-G_{m, t, c} \eta_{f}\right) \\
& \sum_{g \in \Omega_{D g} U \Omega_{T H}} Q_{m, t, g} I_{n, g}-\sum_{l \in\left\{\Omega_{L} \mid v_{l}=n\right\}} Q_{m, t, l}^{r}-\sum_{l \in\left\{\Omega_{L} \mid u_{l}=n\right\}} Q_{m, t, l}^{s}= \\
& +\Psi_{n, t}\left(d_{t, n}-\xi_{m, t, n}^{d}+\xi_{m, t, n}^{c}\right) \\
& +\sum_{c \in\left\{\Omega_{C} \mid n=n_{c}^{a} \text { or } n=n_{c}^{b}\right\}} H_{m, t, c, n}^{Q} \\
& \forall m, t, n \quad(26)
\end{aligned}
$$

The objective function (1) describes the minimization of the discounted expected investment (2) and operational (3) cost. Constraint (4) ensures that the real and reactive power drawn from the grid are within the limits of the substation transformer (i.e. when $g=1$ ). The maximum real and reactive output of DG units is defined in terms of their installed capacity and resource variability as dictated by the timevariable parameter $\zeta_{t, g}$ that captures hourly and seasonal variability.

Constraints (5)-(9) define the state variables that aggregate all investment decisions taken in the past considering their corresponding build times, i.e. the number of epochs between the epoch when the investment decision is made (and the investment cost in (2) is incurred) and the epoch when the investment becomes operational. Constraints (10)-(13) define the real and reactive power flows in polar form. A disjunctive formulation has been implemented to capture the effect that reconductoring has on a line's characteristics $b_{l}$ and $g_{l}$. Different variables are used to model the flow at the sending and receiving ends of each line similar to [22]; differences between these variables represent losses over the line. Constraints (14)-(15) state that real and reactive power flows are bounded by the line's thermal rating.

Constraint (16) imposes the statutory limits on voltage magnitudes across all buses, with the exception of the voltage magnitude at the substation that is defined in (17) to be equal to the voltage target value of the automatic voltage control (AVC) relay of the On Load Tap Changer (OLTC) transformer. This is because when the CVC scheme has not been deployed $\left(\tilde{C}_{m}=0\right)$ then a fixed voltage-target policy for the OLTC at the substation is assumed [23], where $V_{m, t, 1}=$ $V_{m, t}^{n o c}=V_{s e t}$ and $V_{m, t}^{c v c}=0$ according to (17)-(19). If a CVC scheme has been implemented $\left(\tilde{C}_{m}=1\right)$, then the substation voltage target no longer follows a fixed voltage-target policy. Rather, it can be controlled optimally based on real-time information about system voltages; in this case it is $V_{m, t, 1}=$ $V_{m, t}^{c v c}$, with $V_{\text {min }}^{c v c} \leq V_{m, t}^{c v c} \leq V_{\max }^{c v c}$ according to (17)-(19).

Constraints (20) - (22) model the operation of all deployed DSR schemes. In particular, energy equality (20) ensures that all flexible load is eventually served within the period of a typical day; bounds on this load are defined in (21) and (22) respectively. In (21) the load that is disconnected at period $t$ from bus $n$ at node $m$ is zero if no DSR has been deployed at bus $n$ i.e. if $\widetilde{D}_{m, n}=0$. Otherwise, it can attain the maximum value of $f_{t, n} \cdot d_{t, n}$, which is the total amount of flexible load available at time period $t$. The same logic applies for (22).
The SOP installed at NOP $c$ enables the transfer of active power from bus $n_{c}^{a}$ to $n_{c}^{b}$ with efficiency $\eta_{f}$. This transfer has to respect the SOP active power transfer limits according to constraint (23). According to this constraint, if the SOP has not been deployed at $c$ (i.e. $\tilde{S}_{m, c}=0$ ) then the corresponding controlled power flow must be zero i.e. $G_{m, t, c}=0$. Note that SOPs can perform reactive compensation at any of their two terminals. In this regard, constraint (24) imposes the upper bound on the reactive power that a SOP can absorb or generate. Finally, (25) and (26) impose system balance for real and reactive power respectively, while considering actions from smart assets. In (25), the last two terms state that at the bus $n_{c}^{a}$ an amount of $G_{m, t, c}$ is drawn by the SOP and is later released at bus $n_{c}^{b}$, taking into account the efficiency $\eta_{f}$. These two terms do not appear in (26) because SOPs are not capable of transferring reactive power.

\section{CASE STUdY}

We present a case study to demonstrate that the proposed optimization framework can identify the optimal investment strategy that achieves accommodation of DG output at a minimum expected cost under uncertainty in the size, timing and location of DG. In addition, we compare the investment schedules suggested by deterministic and stochastic planning frameworks and illustrate the inherent shortcomings of traditional approaches that do not model uncertainty in an explicit fashion.

\section{A. Description}

Fig. 1 depicts the $11 \mathrm{kV}$ overhead distribution network, based on [24], that is used in the present case study.

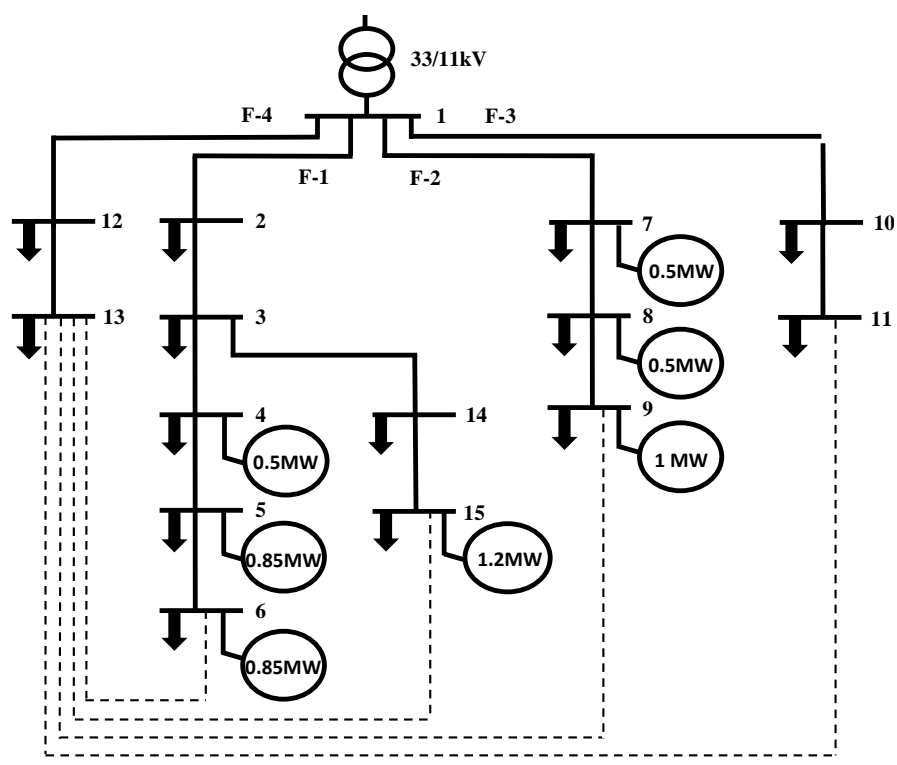

Fig. 1. Schematic diagram of the $11 \mathrm{kV}$ distribution network, showing prospective DG connection points, installed capacities, and all NOPs (dotted lines) corresponding to bus 13. Similar NOPs exist for buses $6,15,9$ and 11, but are not shown for visual clarity purposes.

This network consists of 15 buses, 14 lines and 10 NOPs. Statutory voltage limits at all buses are assumed to be $1.06 \mathrm{pu}$ and $0.94 \mathrm{pu}$ for a base voltage of $11 \mathrm{kV}$. In the first epoch, all 
demand is satisfied through energy imports from the main grid via the primary substation (bus 1). However, an uncertain amount of distributed PV generation is to be connected over the study horizon, which consists of three epochs each of 2year duration. As shown in Fig. 1, there are a total of 7 buses that may accommodate some PV capacity; 4 buses in both Feeder-1 (F-1) and its lateral, and 3 buses in Feeder-2 (F-2). The exact temporal and locational deployment pattern is not known a priori; expert opinion has been utilized to construct the scenario tree shown in Fig. 2 to describe future PV evolution. It consists of 7 nodes comprising 4 scenario paths. Transition probabilities are shown above each arc. Note that for clarity, inside each node we show the aggregate PV capacity installed and the set of buses that the commissioned PV units connect to. Also, above each node we show the value for the voltage target of the AVC relay that controls the OLTC transformer at the primary substation.

The scenario tree used in this case study is shown in Fig. 2; it is assumed to have been constructed following appropriate consultation with developers and system experts. The logic followed in the scenario tree generation is as follows. In the first stage i.e. root node, there is no PV deployed in the system. However, in the subsequent stages there will be PV deployment in either feeder F-1 or F-2. The uncertainty around which one of these two feeders will be fitted with PV is resolved in the second stage transition. In the case of a node $1 \rightarrow$ node 2 transition, buses 5, 6 and 15 on F-1 are fitted with PV. Subsequent transitions in the third stage will determine whether this will remain unchanged (as described by scenario 2 ) or additional PV capacity will be added on bus 4 . Note that the general philosophy is that PV is first built in the most distant buses and may eventually be deployed at buses closer to the substation.

In the absence of CVC, we have assumed that a fixed voltage target policy applies to the AVC relay scheme. In such a case, the voltage target is kept fixed at a value selected traditionally above $1 \mathrm{pu}$ to prevent voltage drops at remote buses [23] and this applies for all scenario-tree nodes as it can be seen in Fig. 2. It has been assumed that this value is changed by the Distribution Network Operator at an interval of one epoch based on the connected PV capacity to the system. For example, those scenario-tree nodes that correspond to higher PV capacity have higher potential for voltage rise and hence the voltage target value is selected to be closer to $1 \mathrm{pu}$ than in other nodes. Under the presence of DG, the fixed voltage target schemes may need to be replaced by CVC schemes which can allow for accurate real-time computation of the voltage target value based on the actual information of voltage magnitudes across the network [23]. It has been assumed that there is no load growth in subsequent epochs and that all buses have identical load profile and magnitude. Also, it is assumed that all load power factors are time-independent and equal to 0.9 .

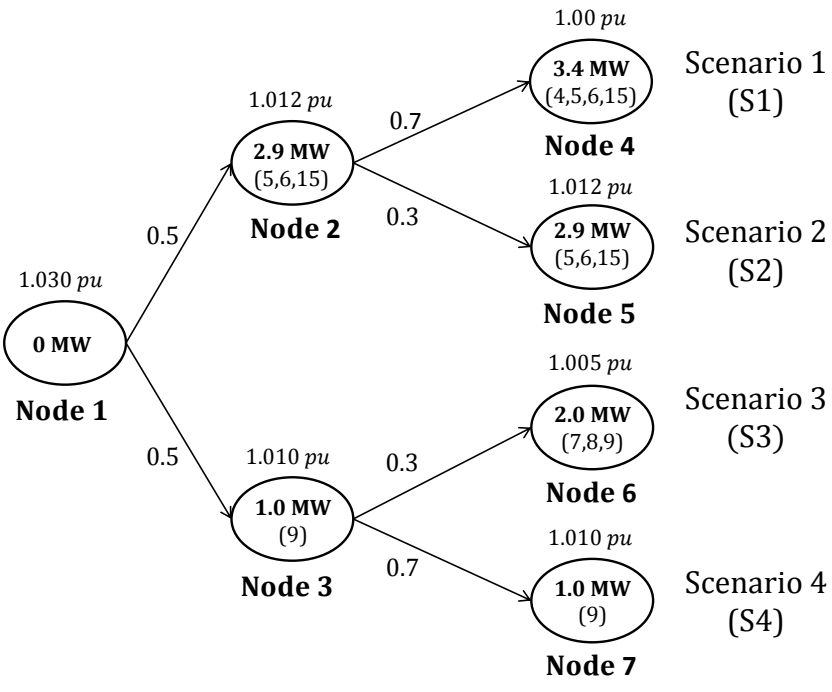

Fig. 2. Scenario tree describing the uncertainty around PV deployment.

The planner has a range of potential solutions for addressing the voltage rise problem, shown in Table I. Note that the APGC of PV units is a service that the PV owners provide to the network operator in exchange for a payment of $£ 100 / \mathrm{MWh}$. The investment cost of the different technologies has been estimated according to relevant sources (see [16] and [25]), while the reasons for the difference in build time between the smart and conventional assets have been described in Section II.

TABLE I. AVAILABLE TECHNOLOGIES FOR INVESTMENT

\begin{tabular}{ccl} 
Technology & Build Time (epochs) & \multicolumn{1}{c}{ Investment Cost (£k) } \\
DSR & 0 & 60 (per bus) \\
CVC & 0 & 540 (whole system) \\
SOP & 0 & 450 (per NOP) \\
Reconductoring & 1 & 320 (per line $\mathrm{km})$ \\
\hline
\end{tabular}

The DSR technology allows the optimal intraday timeshifting of the flexible load, which amounts to $30 \%$ of the total hourly load of each bus. In particular, resolving the voltage rise effect involves shifting the flexible load from periods of small (or zero) PV generation to 'critical' periods of relatively higher PV generation. The resulting effect is the reduction of the net power injections and the restoration of the voltage magnitude within statutory limits.

The CVC technology can measure the actual voltage values at all buses in the network, enabling the optimal regulation of the substation voltage target value. The SOP allows optimal control of the active power flow through its two terminals and optimal reactive compensation at any of its two terminals; 90\% efficiency (in transporting active power from one terminal to the other) with $135 \mathrm{~kW}$ and $135 \mathrm{kVAr}$ capacity is used for these two operations. The reconductoring involves the replacement of the existing conductor with a new line of lower resistance and reactance values. All existing lines have conductors of $35 \mathrm{~mm}^{2}$ with $\mathrm{R} / \mathrm{X}$ factor equal to 2 and resistance equal to $0.9 \Omega / \mathrm{km}$ [26]. The candidate conductor for reconductoring has a cross-sectional area of $300 \mathrm{~mm}^{2}$ with resistance equal to $0.0892 \Omega / \mathrm{km}$ and $\mathrm{R} / \mathrm{X}$ factor equal to 1 . Also, DG units do not perform reactive management (unity power factor operation i.e. $\left.Q_{m, t, g}=0 \forall m, \forall t, \forall g \in \Omega_{D G}\right)$. 
We focus our analysis on 5 typical days, which are used to represent the duration of one year. Each day is characterized by daily patterns for demand and PV output (Fig. 3) corresponding to a particular season and for a location outside London [27]. Since there are four seasons in a year, the use of four typical days would be representative in our analysis. However, since the voltage rise effect is more intense in some periods in the summer than in others, it has been deemed appropriate to consider an extra typical day that corresponds to a hot summer day with relatively higher insolation and lower demand levels than other summer days. The annual frequency of each typical day has been estimated according to [25]; 48 days for the autumn-day and spring-day respectively, 155 days for the winter-day, 70 days for the summer-day and 44 days for the high-summer-day.

Obviously, the greater the number of typical days, the better is the approximation of the annual operational cost. However, with relatively larger number of typical days, the solution times become unacceptably higher. When employing five typical days, the solution times for the stochastic case studies range from half an hour to a little less than a day depending on the number of technologies available to the planner. The increased computational burden is due to the non-linear ACOPF formulation, introduction of inter-temporal constraints to simulate DSR operation and binary variables related to investment decisions.
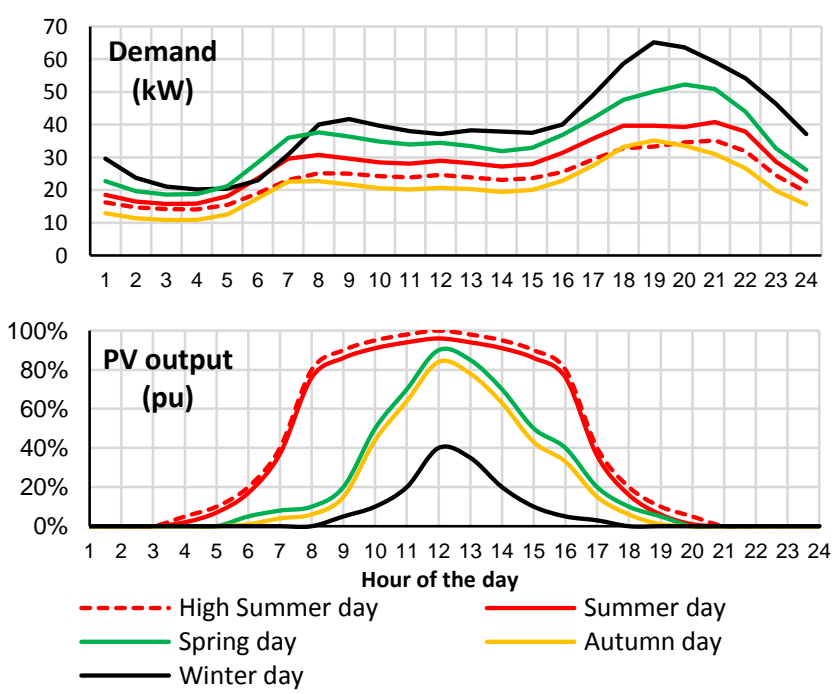

Fig. 3. Patterns per typical day, for demand (above) and generation of a PV unit expressed as a percentage of its installed capacity.

Note that the use of typical days to represent one-year duration is a common approach taken to alleviate the computational load of planning studies without compromising solution integrity (e.g. see [3]). We utilize the model presented in Section III to perform a number of deterministic and stochastic studies. This model includes both thermal and voltage constraints. In the case study we have selected a value of $F_{l}$ (thermal capacity of the existing lines) that is sufficiently high so that the system is bounded only by voltage constraints.

All models were developed using FICO Xpress 7.8 and all studies were carried out on a Xeon $3.46 \mathrm{GHz}$ computer. For the solution of the MINLP that arises, the "mmxnlp" module of the Xpress-NLP optimization engine has been used. This solution strategy involves a combination of Sequential Linear Programing (SLP) to approximate non-linear elements and a traditional Branch and Bound (B\&B) technique to identify optimal value of binary variables. Multiple SLP optimisations on the integer-relaxed problem are first carried out to identify candidate solutions and avoid entrapment to a local minimum. Subsequently, each candidate is used as the initial seed for a B\&B search; sub-optimal search paths are progressively discarded until a superior solution emerges. Although an exhaustive search is necessary to guarantee a global optimum, extensive studies were carried out with different engine settings to ensure comprehensive exploration of the solution space. It is important to highlight that it is possible to simplify the quadratic constraints (4), (14) and (15) by replacing them with box constraints as demonstrated in [28]. As expected, this relaxation leads to improved computational performance at the expense of introducing technically infeasible operating points in the solution space. In the present case study, the full quadratic formulation has been used.

\section{B. Deterministic planning}

Deterministic planning involves finding the optimal investment schedule for each of the four scenarios $(\mathrm{S} 1-\mathrm{S} 4)$ by applying the model described in Section III. The optimal investment plans are displayed in Fig. 4. The following notation applies: $\mathrm{D}(\mathrm{n})$ represents the decision to invest in DSR at bus $n, \mathrm{SOP}(\mathrm{a}-\mathrm{b})$ represents the decision to invest in a SOP at the NOP of buses a and $b$, while CVC denotes the decision for installation of a CVC scheme in the substation. The [a-b] represents the decision to invest in reconductoring of line a b. Note that lines become operational one epoch after the investment decision is made. Table II presents information on the total cost (denoted $\mathrm{T}$ Cost), which is the summation of investment cost (I Cost) and operation cost (O Cost), as well as the percentage of curtailed energy produced from PVs (PV Curt) per scenario.

All four investment plans are dominated by decisions to undertake conventional rather than smart investments, with the majority of commitments made in the first epoch. Despite the availability of DSR, SOP and CVC technologies, they are deemed unattractive. Regarding APGC, the scenarios S1 - S2 which involve large amount of conventional reinforcements involve less APGC than S3 - S4 where the opposite applies. This happens because the objective involves the minimization of the investment and operational costs, so that when there are few available lines to reconductor (as it is the case in S3-S4), APGC service is used as a complement in the attempt to tackle the voltage rise in the most economical way possible. Note that if the APGC technology and the rest smart technologies (CVC, DSR, SOP) are not available to the planner, then the resulting investment plans are very similar to those in Fig. 4; the first two scenarios include two more first-stage investment decisions ([5-6], [3-14]) while the last two include two investment decisions in the first stage ([1-7], [8-9]). Hence, for a deterministic planner, the presence of CVC, SOP and DSR has no impact on the optimal investment plan since there is no 
need for flexibility-driven investments; exploitation of economies of scale is the most important driver instead.

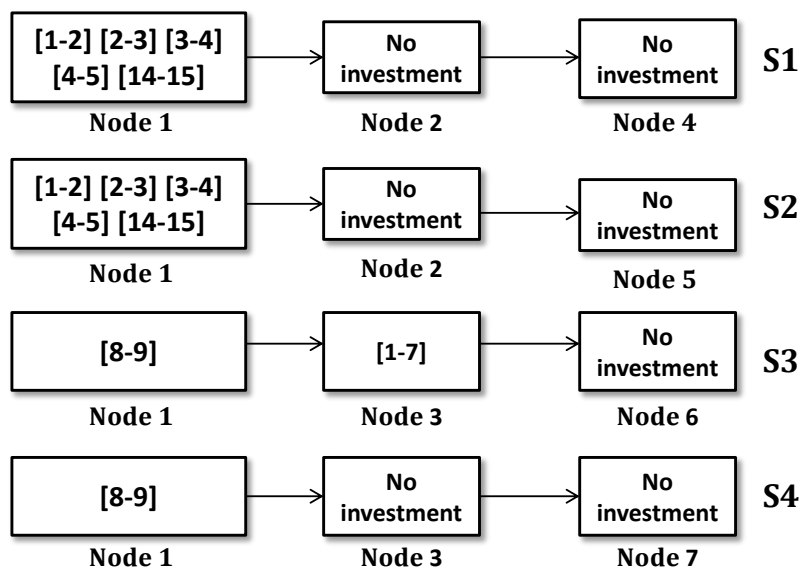

Fig. 4. Optimal investment plans when each scenario is solved individually and all technologies are available to the planner.

TABLE II. DETERMINISTIC STUDY RESULTS

\begin{tabular}{llccc}
\cline { 2 - 5 } & T Cost & I Cost & O Cost & PV Curt \\
\hline S1 & $£ 478.9 \mathrm{k}$ & $£ 454.9 \mathrm{k}$ & $£ 23.9 \mathrm{k}$ & $2.5 \%$ \\
S2 & $£ 501.0 \mathrm{k}$ & $£ 455.9 \mathrm{k}$ & $£ 45.1 \mathrm{k}$ & $5.0 \%$ \\
S3 & $£ 168.7 \mathrm{k}$ & $£ 148.5 \mathrm{k}$ & $£ 20.2 \mathrm{k}$ & $6.0 \%$ \\
S4 & $£ 129.4 \mathrm{k}$ & $£ 90.6 \mathrm{k}$ & $£ 38.8 \mathrm{k}$ & $12.0 \%$ \\
\hline
\end{tabular}

\section{Stochastic planning}

Stochastic planning involves identifying the optimal investment strategy that results in the minimization of the expected system cost across the horizon. In this section three different studies are carried out. In the first study, line reconductoring is the sole available investment alternative and firm DG connections apply i.e. no APGC is allowed. In the second study, line reconductoring is the sole available investment alternative while APGC is allowed. In the last study, the planner can consider all conventional and smart technologies, including APGC.

The optimal investment strategies are shown in Fig. 5, Fig. 6 and Fig. 7 respectively. In the same manner, Table III, Table IV and Table $\mathrm{V}$ present the corresponding information on investment and operation costs as in Table II; an extra row that displays expected values (indicated by E) has also been added. By comparing these investment strategies, we can observe that the availability of smart technologies to the stochastic planner radically affects both the timing and the type of the optimal investment decisions. In particular, the strategy presented in Fig. 7 and Table $\mathrm{V}$ involves reduced total expected system cost, far fewer decisions to invest in conventional assets, high deployment of smart grid assets and no first-stage capital commitments.

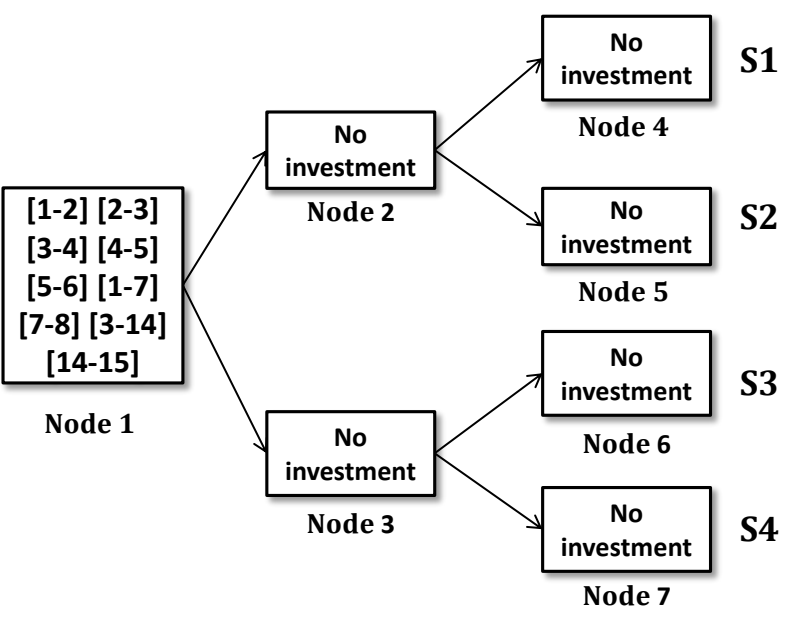

Fig. 5. Solution strategy for a stochastic planner with only reconductoring available for investment. Note that APGC is not available.

TABLE III. COSTS AND PV CURTAILMENT FOR THE INVESTMENT STRATEGY WITHOUT SMART TECHNOLOGIES AND WITHOUT APGC

\begin{tabular}{clccc} 
& T Cost & I Cost & O Cost & PV Curt \\
\hline S1 & $£ 818.0 \mathrm{k}$ & $£ 818.0 \mathrm{k}$ & $£ 0 \mathrm{k}$ & $0 \%$ \\
S2 & $£ 818.0 \mathrm{k}$ & $£ 818.0 \mathrm{k}$ & $£ 0 \mathrm{k}$ & $0 \%$ \\
S3 & $£ 818.0 \mathrm{k}$ & $£ 818.0 \mathrm{k}$ & $£ 0 \mathrm{k}$ & $0 \%$ \\
S4 & $£ 818.0 \mathrm{k}$ & $£ 818.0 \mathrm{k}$ & $£ 0 \mathrm{k}$ & $0 \%$ \\
\hline E & $£ 818.0 \mathrm{k}$ & $£ 818.0 \mathrm{k}$ & $£ 0 \mathrm{k}$ & $0 \%$ \\
\hline
\end{tabular}

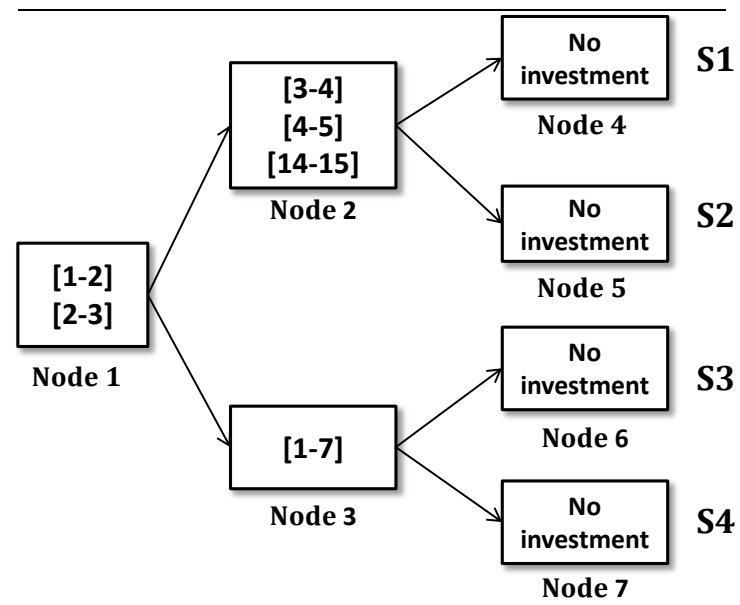

Fig. 6. Solution strategy for a stochastic planner with only reconductoring available for investment. APGC service is also available.

TABLE IV. COSTS AND PV CURTAILMENT FOR THE INVESTMENT STRATEGY WITHOUT SMART TECHNOLOGIES AND WITH APGC

\begin{tabular}{clccc} 
& T Cost & I Cost & O Cost & PV Curt \\
\hline S1 & $£ 666.7 \mathrm{k}$ & $£ 354.6 \mathrm{k}$ & $£ 312.1 \mathrm{k}$ & $14.4 \%$ \\
S2 & $£ 660.1 \mathrm{k}$ & $£ 354.6 \mathrm{k}$ & $£ 305.5 \mathrm{k}$ & $16.0 \%$ \\
S3 & $£ 379.6 \mathrm{k}$ & $£ 239.4 \mathrm{k}$ & $£ 140.2 \mathrm{k}$ & $14.2 \%$ \\
S4 & $£ 339.4 \mathrm{k}$ & $£ 239.4 \mathrm{k}$ & $£ 100.0 \mathrm{k}$ & $14.8 \%$ \\
\hline E & $£ 508.1 \mathrm{k}$ & $£ 242.5 \mathrm{k}$ & $£ 211.1 \mathrm{k}$ & $14.8 \%$ \\
\hline
\end{tabular}




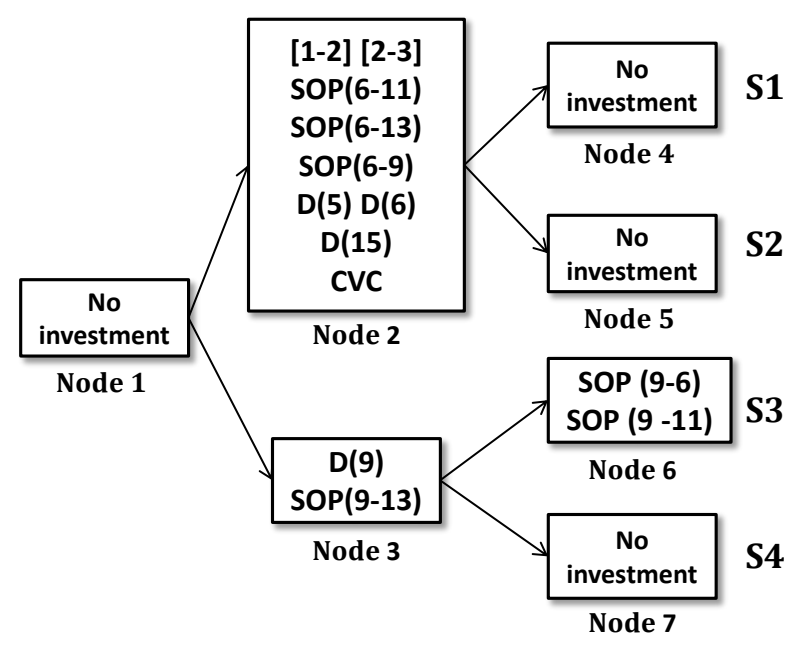

Fig. 7. Solution strategy for a stochastic planner with all technologies (SOP, DSR, CVC, reconductoring) available for investment. APGC service is also available.

TABLE V. COSTS AND PV CURTAILMENT FOR THE INVESTMENT STRATEGY WITH SMART TECHNOLOGIES AND APGC

\begin{tabular}{clccc}
\cline { 2 - 5 } & T Cost & I Cost & O Cost & PV Curt \\
\hline S1 & $£ 742.2 \mathrm{k}$ & $£ 488.7 \mathrm{k}$ & $£ 253.4 \mathrm{k}$ & $11.8 \%$ \\
S2 & $£ 721.5 \mathrm{k}$ & $£ 488.7 \mathrm{k}$ & $£ 232.8 \mathrm{k}$ & $11.7 \%$ \\
S3 & $£ 207.1 \mathrm{k}$ & $£ 168.9 \mathrm{k}$ & $£ 38.2 \mathrm{k}$ & $3.9 \%$ \\
S4 & $£ 128.9 \mathrm{k}$ & $£ 91.8 \mathrm{k}$ & $£ 37.1 \mathrm{k}$ & $5.6 \%$ \\
\hline E & $£ 444.2 \mathrm{k}$ & $£ 301.8 \mathrm{k}$ & $£ 142.4 \mathrm{k}$ & $8.4 \%$ \\
\hline
\end{tabular}

When uncertainty is considered, smart technologies like CVC and SOPs constitute attractive investment opportunities due to the operational flexibility they can provide. Fig. 8 illustrates an example of CVC operation, where a voltage violation at bus 6 is resolved by controlling the substation voltage during the time of high PV output. This is a clear demonstration of CVC's ability to alleviate constraints at different parts of the network through enhanced controllability. The benefit of CVC's control capabilities persist under all scenarios of future DG connections, whereas re-conductoring is subject to stranding if the envisaged scenario does not materialise.

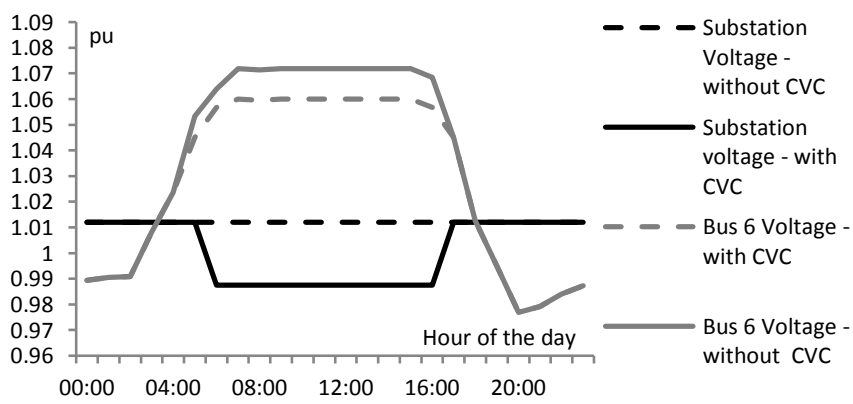

Fig. 8. Impact of CVC operation on the substation voltage bus 6 at scenario-tree-node 2 , high summer day.

The basic operating principle of the SOP technology for tackling the voltage rise effect is depicted in Fig. 9. The voltage magnitude at bus 6 is kept within statutory limits by a combination of absorption of reactive power (shown negative) at bus 6 and active power transfer from bus 6 to bus 9 during hours of high PV generation. SOP is shown to enhance flexibility towards managing the active/reactive power balance between adjacent feeders. As such, it is much less susceptible to stranding risks when compared to re-conductoring which solely increases power transfer capability between two points.

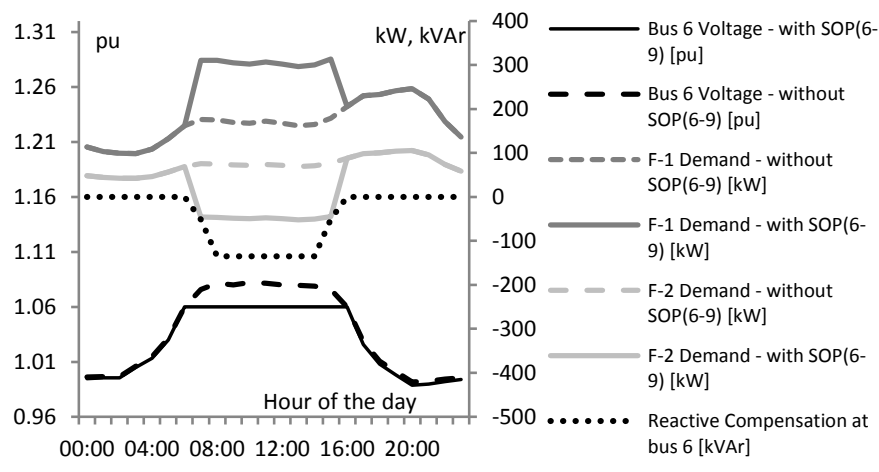

Fig. 9. Demonstration of the operation of the SOP deployed at NOP 6-9 at scenario-tree node 4, high-summer day.

\section{Strategic value of smart grid technologies}

Smart grid assets offer significant strategic benefits when planning under uncertainty due to their fast commissioning time and the enhanced network controllability they can provide at different parts of the network which renders them robust against a wide range of scenarios. Of course, relying solely on smart assets may not be possible to alleviate all constrains, but the ability to deploy them in a contingent fashion can be very useful in 'buying time' in the interim until conventional large-volume reinforcements can become operational. For example, by comparing between Fig. 5 and Fig. 7, it is evident that the availability of smart assets enables the adoption of a 'wait-and-see' strategy and relieves the need for first-stage commitments which are highly susceptible to stranding risks prior to the resolution of locational uncertainty. Although once the new conductors become operational the need for smart asset controllability is reduced, meaning that some over-investment has essentially taken place, the adoption of a 'wait-and-see' strategy would not have been possible without them facilitating operation in the interim. This point is reinforced by contrasting to the deterministic case; as shown in Fig. 4, smart grid assets are not attractive in the absence of uncertainty, due to the limited scope for strategic investment.

By comparing between the total expected cost of the strategies shown in Fig. 5 and Fig. 7 we can quantify the strategic value of the portfolio of smart technologies consisting of CVC, APGC, SOP and DSR. This value amounts to $£ 818 \mathrm{k}-£ 444.2 \mathrm{k}=£ 373.8 \mathrm{k}$ i.e. $45 \%$ cost reduction and represents the expected savings in terms of investment and operation costs due to the ability to invest in smart grid assets. If APGC is available in the basecase (i.e. comparing Fig. 6 to Fig. 7), strategic value of CVC, SOP and DSR is equal to $£ 63.9 \mathrm{k}$, corresponding to a $13 \%$ cost reduction. Note that APGC does not entail direct investment costs but can be interpreted as a policy decision to curtail renewable energy output for a penalty when needed. Similarly, we can quantify the strategic value of each different technology. 
In Fig. 10 we illustrate the strategic value of each of the four smart grid technologies; APGC, CVC, SOP, and DSR as a function of the availability of other technologies. This type of analysis allows us to quantify the magnitude of strategic benefit entailed in each as well as assess the degree of complementarity between them.

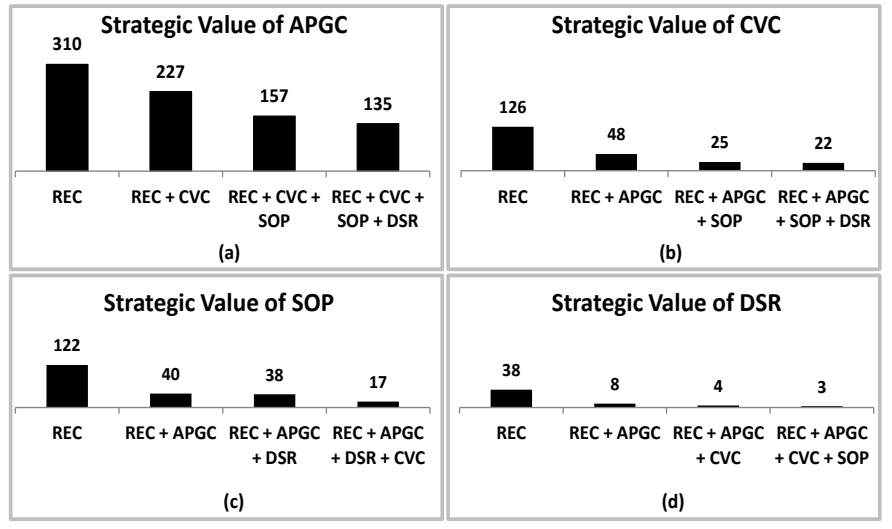

Fig. 10. Strategic Value (£k) of a smart technology as a function of the availability of other technologies (REC refers to reconductoring).

As expected, the strategic value of a technology reduces as more smart technologies become available. For the specific case study, the most valuable technology is APGC, followed by CVC, SOP and DSR. In particular, APGC is shown to entail considerable strategic value since it enables costbeneficial PV curtailment across the entire network and carries no investment cost. CVC and SOP are also highly valuable due to their ability to increase network controllability across the entire network and across adjacent feeders respectively. DSR is shown to embed comparatively low levels of strategic flexibility due to fact that it has to be targeted at a specific bus, thus entailing more pronounced stranding risks.

\section{CONCLUSION AND FUTURE WORK}

This paper proposes a model for identifying the optimal investment strategy towards alleviating network constraints that may arise due to uncertain DG penetration. The model considers investment in reconductoring and various smart technologies (SOP, CVC, DSR, and APGC). Through a case study on an $11 \mathrm{kV}$ network, we demonstrate that smart technologies considerably alleviate the need for anticipatory conventional commitments as the planner can take advantage of the strategic flexibility embedded in such technologies; application of conventional deterministic approaches systematically undervalues this benefit and may lead to unnecessarily high levels of risks of stranded assets. The inherent flexibility of smart technologies is crystallized in the concept of strategic value. An important insight of the present paper is that when adopting a planning framework aimed at accommodating long-term uncertainty at least cost, and capturing strategic value, fundamentally different network designs emerge. Flexible smart grid technologies are favored in cases where they aid with future system adaptability, while investment in conventional assets is postponed until it can be justified on the basis of the observed uncertainty evolution. The increasing uncertainty in combination with the lack of regulatory frameworks that consider a candidate investment's strategic value is bound to hinder the deployment of smart grid assets and favor long-term capital commitments at the detriment of flexibility. The presented methods can be instrumental in enabling a shift towards strategic planning.

The focus of our future work lies in the development and application of decomposition and linearization techniques for achieving more efficient solution times as outlined in the recent literature (e.g. see [29] and [30]). Development of novel solution strategies is also seen as a fundamental step towards achieving more efficient solution times. In particular, the combination of temporal decomposition with tight relaxation schemes has already been demonstrated to offer impressive computational gains in the context of stochastic transmission planning [31]. Extending this scheme to the non-linear ACOPF paradigm will be imperative for rendering the proposed modelling approach scalable to larger systems and scenario trees. Novel online convex optimization-based algorithms presented in [32] could also be investigated for improving problem tractability. Another future topic constitutes the investigation of risk-averse decision criteria (such as CVaR) to capture the planners' attitude towards adverse scenario realizations. Future research will involve the consideration of a wider range of conventional reinforcement options. For example, different conductor types, each with its own technical characteristics and cost profile, different capacitor types and other forms of compensation could be considered for network reinforcement. In addition, a greater number of smart technologies can be included in the formulation, such as dynamic line rating. Other sources of system flexibility can also be exploited; researchers in [33] show that capabilities of power inverters can be exploited for voltage regulation. Finally, another area for improvement is the consideration of uncertainty at operating timescales. In this regard, of most interest is modelling demand level uncertainty which can have an impact on the utilization of DSR resources.

\section{REFERENCES}

[1] Eurelectric, Active Distribution System Management - Full Discussion Paper, Feb. 2013.

[2] O. Brewin, S.C.E. Jupe, M. G. Bartlett, K.T. Jackson and C. Hanmer, "New Technologies for Low Voltage Distribution Networks", ISGT Europe, Manchester, 2011.

[3] S.N. Liew and G. Strbac, "Maximizing Penetration of Wind Generation in Existing Distribution Networks", IEE Proc. on Gen., Trans. and Distrib., vol. 149, no. 3, 2002.

[4] J.M. Bloemink and T.C. Green, "Benefits of distribution-level power electronics for supporting distributed generation growth", IEEE Trans. Power Del., vol. 28, no.2, pp. 911-919, Apr. 2013.

[5] J. Yi, P. Wang, P. C. Taylor, P. J. Davison, P. F. Lyons, D. Liang, S. Brown and D. Roberts, "Distribution network voltage control using energy storage and demand side response", ISGT Europe, Berlin, 2012.

[6] G. Strbac, "Demand side management: Benefits and challenges," Energy Policy, vol. 36, no. 12, pp. 4419-4426, 2008.

[7] G. Strbac, I. Konstantelos, M. Pollitt, R. Green, Delivering future-proof energy infrastructure: Report for National Infrastructure Commission, Feb. 2016.

[8] A. Keane, L. F. Ochoa, C. L. T. Borges, G. W. Ault, A. D. AlarconRodriguez, R. A. F. Currie, F. Pilo, C. Dent and G. P. Harrison, "Stateof-the-art techniques and challenges ahead for distributed generation planning and optimization", IEEE Trans. Power Syst., vol. 28, no. 2, pp. 1493-15012, May 2013. 
[9] S. You, H. W. Bindner, J. Hu and P. J. Douglass, "An overview of trends in distribution network planning: A movement towards smart planning", T\&D Conference and Exposition, 2014 IEEE PES, Chicago, pp. 1-5, 2014.

[10] P. C. Paiva, H. M. Khodr, J. A. Dominguez-Navarro, J. M. Yusta, and A. J. Urdaneta, "Integral planning of primary-secondary distribution systems using mixed integer linear programming," IEEE Trans. Power Syst., vol. 20, no. 2, pp. 1134-1143, May 2005.

[11] S. Haffner, L. F. A. Pereira, L. A. Pereira, and L. S. Barreto, "Multistage model for distribution expansion planning with distributed generation-part I: problem formulation," IEEE Trans. Power Del., vol.23, no. 2, pp. 915-923, Apr. 2008.

[12] P. S. Georgilakis and N. Hatziargyriou, "Optimal distributed generation placement in power distribution networks: models, methods, and future research", IEEE Trans. Power Syst., vol. 28, no. 3, Aug. 2013.

[13] E. Buzarquis, G. A. Blanco, F. Olsina, and F. F. Garces, "Valuing investments in distribution networks with DG under uncertainty," $T \& D$ Conference and Exposition: Latin America, 2010 IEEE PES, Sao Paulo, pp. 341-348, 2010.

[14] I. Konstantelos and G. Strbac, "Valuation of flexible investment options under uncertainty", IEEE Trans. Power Syst., vol. 30, no. 2, pp. 1047-1055, Mar. 2015.

[15] B. A. Weisbrod, "Collective-consumption services of individualconsumption goods", The Quarterly Journal of Economics, vol. 78, no. 3, pp. 471-477, 1964.

[16] Frontier Economics, A framework for the evaluation of smart grids: A report prepared for Ofgem, Mar. 2012.

[17] Ofgem, Real Options and Investment Decision Making, Mar. 2012.

[18] F. Capitanescu, "Critical review of recent advances and further developments needed in AC optimal power flow", Elec. Power Syst. Res., vol. 136, pp. 57-68, 2016.

[19] L. Che, X. Zhang, M. Shahidehpour, A. Alabdulwahab, A. Abusorrah, "Optimal Interconnection Planning of Community Microgrids With Renewable Energy Sources," IEEE Trans. on Smart Grid, vol. PP, no. 99, pp.1-10.

[20] A.J. Conejo, M. Carrion, and J.M. Morales, Decision making under uncertainty in electricity markets, New York: Springer, 2010.

[21] I. Konstantelos, "A stochastic optimization framework for anticipatory transmission investment," Ph.D. dissertation, Department of Electrical and Electronic Engineering, Imperial College London, 2012.

[22] X. Zhang, G. Karady, K. Piratla, and S. Ariaratnam, "Network capacity assessment of combined heat and power-based distributed generation in urban energy infrastructures," IEEE Trans. Smart Grid, vol. 4, no. 4, pp. 2131-2138, Dec. 2013.

[23] M. Fila, D. Reid, G. A. Taylor, P. Lang, and M. R. Irving, "Coordinated voltage control for active network management of distributed generation," IEEE PES Gen. Meeting, Calgary, pp.1-8, Jul. 2009.

[24] H. M. Khodr, F. G. Olsina, P. M. Oliveira-De Jesus, and J.M. Yusta, "Maximum savings approach for location and sizing of capacitors in distribution systems," Elec. Power Syst. Res., vol. 78, no. 7, pp. 11921203, 2008.

[25] Elexon. Load profiles and their use in electricity settlement, version 2.0, Nov. 2013.

[26] E.ON, Network Design Manual v7.7, Dec. 2006.

[27] I. Konstantelos, M. Sun and G. Strbac, Quantifying demand diversity of households, Report for the "Low Carbon London" LCNF project, Imperial College London, 2014.

[28] R. D. Zimmerman, C. E. Murillo-Sanchez, and R. J. Thomas, "Matpower: steady state operations, planning and analysis tools for power systems research and education," IEEE Trans. Power Syst., vol. 26, no. 1, pp. 12-19, Feb. 2011

[29] R. S. Ferreira, C. L. T. Borges and M. V. F. Pereira, "A Flexible Mixed-Integer Linear Programming Approach to the AC Optimal Power Flow in Distribution Systems," IEEE Trans. Power Syst., vol. 29, no. 5, pp. 2447-2459, Sep. 2014.

[30] C. Coffrin, P. Van Hentenryck, and R. Bent, "Approximating line losses and apparent power in AC power flow linearizations", IEEE PES Gen. Meeting, San Diego, CA, 2012, pp. 1-8, Jul. 2012.

[31] P. Falugi, I. Konstantelos and G. Strbac, "Application of novel nested decomposition techniques to long-term planning problems," accepted for presentation in PSSC '16, Genoa, 2016.

[32] V. Kekatos, G. Wang, A.-J. Conejo, and G. B. Giannakis, "Stochastic reactive power management in microgrids with renewables," IEEE Trans Power Syst., vol. 30, no. 6, pp. 3386-3395, November 2015.
[33] G. Wang, V. Kekatos, A. J. Conejo, and G. B. Giannakis, "Ergodic energy management leveraging resource variability in distribution grids," http://arxiv.org/abs/1508.00654, Aug. 2015.

\section{BIOGRAPHIES}

Ioannis Konstantelos received the MEng degree in Electrical and Electronic Engineering from Imperial College London in 2007. He obtained his PhD from the same university in 2013 in the field of electrical energy systems. His research interests include mathematical programming and statistical modeling techniques applied to the planning and operation of energy systems.

Spyros Giannelos received the MEng degree in Electrical and Electronic Engineering from National Technical University of Athens in 2009. His research interests include distribution network investment planning and operation and uncertainty modeling applied to electricity infrastructure. He is currently a PhD candidate at Imperial College London.

Goran Strbac is a Professor of electrical energy systems at Imperial College, London, UK. His current research interests include electricity generation, transmission and distribution operation, planning and pricing, and integration of renewable and distributed generation in electricity systems. 